\title{
Parameter estimation of K-distributed sea clutter based on fuzzy inference and Gustafson-Kessel clustering
}

\begin{abstract}
The detection performance of maritime radars is restricted by the unwanted sea echo or clutter. Although the number of these target-like data is small, they may cause false alarm and perturb the target detection. K-distribution is known as the best fit probability density function for the radar sea clutter. This paper proposes a novel approach to estimate the parameters of K-distribution, based on fuzzy Gustafson-Kessel clustering and fuzzy TakagiSugeno Kang modelling. The main contribution of the proposed method is the ability to estimate the parameters, given a small number of data which will usually be the case in practical applications. This is achieved by a pre-estimation using fuzzy clustering that provides a prior knowledge and forms a rough model to be fine tuned using the least square method. The algorithm also improves the calculations of shape and width of membership functions by means of clustering in order to improve the accuracy. The resultant estimator then acts to overcome the bottleneck of the existing methods in which it achieves a higher performance and accuracy in spite of small number of data.
\end{abstract}

Keyword: K-distribution; Parameter estimation; Fuzzy GK-clustering; Fuzzy TSK modelling. 